Joachim De Stercke Gaëtan Temperman

Université de Mons (Belgique)

\section{Le sentiment d'auto-efficacité des pompiers en matière de formation}

\author{
Firefighters'Self-efficacy in Training and Instruction
}

doi: $10.18162 / f p .2021 .590$

\section{ésumé}

Cette étude analyse limpact de la formation pédagogique sur le sentiment d'auto-efficacité de pompiers en matière de formation. Pour ce faire, 125 pompiers belges ont été interrogés via un questionnaire dérivé de la Teachers'Sense of Efficacy Scale avant et après leur participation à un module pédagogique. Notre étude montre qu'au terme de celui-ci, ils se sentent globalement plus efficaces (effet d'apprentissage), mais aussi que les disparités inter-sujets tendent à se réduire (effet d'équité). II en ressort également que le parcours ou l'expérience pédagogique acquise antérieurement par certains participants influence leur sentiment d'auto-efficacité.

Mots-clés

Sentiment d'auto-efficacité, Sentiment d'efficacité personnelle, Pompier, Formation pédagogique, Croyances d'efficacité.

Abstract

This study analyses the impact of instructor training on firefighters' self-efficacy in education and training. Overall, 125 firefighters participated to a questionnaire survey using an adaptation of the Teachers' Sense of Efficacy Scale before and after having completed an instructor training module. At the end of training, they do feel more

efficient (learning effect). Also, in betweensubjects variation of self-efficacy tends to decrease (equity effect). Moreover, firefighters' background and prior experiences in education and training do influence their self-efficacy.

Keywords

Self-efficacy, Firefighter, Instructor Training, Training, Efficacy beliefs.

\section{Introduction}

La plupart des recherches conduites sur le sentiment d'auto-efficacité des pompiers traitent de la santé physique et psychologique de cette catégorie professionnelle exposée à de nombreux risques. Parmi celles-ci, on retrouve des travaux en rapport au coping et à résilience, à l'anxiété et au stress, au burnout ou encore au stress post-traumatique (Deppa et Saltzberg, 2016; Lambert, Benight, Harrison et Cieslak, 2012; Markra-Studzinka, Golonka et Izydorczyk, 2019). Bien que la formation constitue l'un des principaux piliers de la réforme de la sécurité civile belge (2007), peu d'études s'y sont à ce jour intéressées. La formation pédagogique des pompiers susceptibles d'assurer la fonction d'instructeur n'a pas fait l'objet de plus d'attention. Cet article propose de pallier ce manque en analysant les croyances d'efficacité en matière de formation des pompiers avant et après avoir complété un module pédagogique. Ce faisant, nous étudierons également la structure factorielle du questionnaire conçu pour l'occasion ainsi que les variables qui pourraient expliquer les différences de sentiment d'auto-efficacité entre répondants.

\section{La formation pédagogique des pompiers belges}

En Belgique, la formation des membres des services publics de secours est régie par l'Arrêté royal (AR) du 18 novembre 2015. Ce texte distingue trois types de formations : la formation de base (composée des brevets liés à l'entrée en carrière et à l'évolution hiérarchique), la formation continue (visant à compléter ou améliorer les compétences des intervenants) et la formation permanente (consistant à maintenir leurs acquis). Les deux premières relèvent de la responsabilité des centres de formation (ou «école du feu)» agréés par le Ministre de l'Intérieur et la dernière des zones de secours ${ }^{1}$. La formation 
continue, que les pompiers doivent suivre à raison d'un minimum de $120 \mathrm{~h}$ sur cinq ans, regroupe deux types de formations : les attestations et les certificats. Ces derniers ont la particularité d'être liés à une fonction spécialisée (e.g. plongeur). Pour remplir ses missions, tout centre de formation a l'obligation de comporter une cellule pédagogique composée d'experts opérationnels et d'un pédagogue.

Conformément à l'AR «Formation» et à l'Arrêté ministériel (AM) relatif à la formation et au certificat FOROP1 et FOROP2 dit «FOROP» (2019), la formation pédagogique des pompiers s'articule autour de deux modules. Le FOROP1 (40h) constitue le premier niveau de qualification auquel les pompiers peuvent prétendre. Sa finalité est de les rendre aptes à dispenser des cours sur base d'un programme préétabli et à l'aide d'outils (fiche pédagogique, cartes d'exercices, etc.) mis à leur disposition via une «valise pédagogique». Le FOROP2 (40h), soit le deuxième niveau de qualification atteignable, vient compléter les compétences pédagogiques des pompiers en mettant l'accent sur la maîtrise des bases légales de la formation, sur la conception des valises pédagogiques et l'utilisation des outils technopédagogiques ainsi que sur le management d'équipes d'instructeurs. Ces deux modules peuvent être entrepris soit dans le cadre de la formation de base (M01 pour le FOROP1 et OFF2 pour le FOROP2 $2^{2}$ ), soit dans le cadre de la formation continue (certificat associé à une obligation de recyclage de $24 \mathrm{~h}$ tous les dix ans). Tous les pompiers professionnels aspirant à devenir Sergent sont tenus d'obtenir le FOROP1. Pour les pompiers volontaires, il s'agit d'un module optionnel. Depuis janvier 2021, le FOROP1 est obligatoire pour dispenser des formations de base ou continues; les formations pédagogiques étant dispensées par des instructeurs possédant un diplôme en pédagogie ou à défaut détenteurs du FOROP2, sous la tutelle de leur cellule pédagogique. Avant la réforme, les formations pédagogiques se déclinaient en cinq modules : FOROP1 (8h), FOROP2 (40h; équivalent au FOROP1 post-réforme), FOROP3 (40h; jugé un temps équivalent au FOROP2 post-réforme bien que moins complet), module Instructeur du brevet d'Officier par promotion (40h) et module Instructeur du brevet d'Officier par recrutement (40h). Ces deux derniers modules équivalents au FOROP3 couvraient les mêmes contenus et objectifs.

La réforme de la formation a entrainé une nette augmentation du volume d'heures - surtout pratiques - à organiser pour les centres de formation, avec pour conséquence la massification de leur pool d'instructeurs. Plusieurs problématiques émergent de cette situation. L'attractivité de la fonction d'instructeur et leur recrutement par les centres de formation, qui sont des employeurs distincts des zones de secours, en est une. Elle touche de manière variable les écoles du feu du pays. Le centre de formation dont il est question dans cet article, situé en province de Hainaut, n'en souffre pas. Il s'agit d'un centre de taille importante au sein duquel le recrutement des instructeurs est formalisé et structuré autour d'une procédure qualité (ISO9001). Cette procédure passe par un appel à candidatures, un entretien individuel puis par une observation in situ à l'occasion d'un stage probatoire avant que le candidat accède à la fonction, sa reconduction restant soumise à une évaluation annuelle. Avec près de $10 \%$ des pompiers hennuyers exerçant la fonction d'instructeur accessoirement à leur mission opérationnelle ${ }^{3}$, c'est davantage avec le problème de leur disponibilité que doivent composer les partenaires de la formation dans cette province, car, faut-il le noter, la massification des forces de travail, dans le contexte qui nous occupe, augmente le risque de recruter des pompiers moins intéressés par la fonction d'instructeur pour des raisons intrinsèques ou altruistes que pour ses avantages extrinsèques (dont la rémunération). Au-delà des facteurs motivationnels, la question de l'aptitude à exercer efficacement la fonction devient, elle aussi, critique. Comment assurer la qualité et l'harmonisation des formations

2 - Formation et profession 29(2), 2021 
avec un pool d'instructeurs grandissant et face à l'inexorable complexification de la profession? Afin de réduire les risques afférents à cette question, des formations de formateurs ont vu le jour en référence aux différents domaines de compétence des pompiers, en complément des formations pédagogiques.

\section{Le sentiment d'auto-efficacité}

Le concept de sentiment d'efficacité personnelle, ou sentiment d'auto-efficacité, renvoie dans sa dimension individuelle (Hoy et Spero, 2005) au jugement que porte un individu sur sa capacité à effectuer une action particulière en vue d'atteindre un objectif qu'il s'est fixé (Bandura, 2003). Le sentiment d'auto-efficacité est une perception subjective qui n'est par nature pas toujours rationnelle ou en rapport avec les capacités réelles de l'individu. Dans l'estimation de son aptitude à accomplir une tâche dans une situation donnée, celui-ci évalue, outre ses capacités, le niveau d'exigence attendu, les conditions spécifiques de réalisation de cette tâche ou encore les ressources qu'il peut mobiliser pour l'accomplir. Les sources de ce sentiment sont de quatre ordres : les expériences antérieures de maîtrise (ré)interprétées par l'individu, les expériences vicariantes, la persuasion verbale et les états physiologiques et émotionnels. À cela s'ajoutent les sources comportementales qui naissent au confluent de l'individu et de l'environnement. Parmi les issues mises au jour par Bandura (2003), on retiendra que les apprenants possédant un sentiment d'efficacité personnelle élevé s'engagent davantage, sont plus persévérants et plus performants face à une tâche à réaliser. Dans le domaine de la formation des enseignants, Pfitzner-Eden (2016) met en évidence que le sentiment d'efficacité personnelle constitue une variable dépendante à considérer dans le cadre de l'analyse de l'effet de la formation. Les résultats de cette étude montrent que les changements d'efficacité personnelle perçue des futurs enseignants sont significativement prédits par leurs expériences de maîtrise; ceux-ci révèlent également l'influence des trois autres sources du concept.

En ce qui concerne la mesure, Tschannen-Moran et Woolfolk-Hoy (2007) proposent d'analyser le sentiment d'efficacité personnelle des enseignants à l'aide de trois grandes dimensions : l'engagement des apprenants, les stratégies d'enseignement et la gestion de classe. Chez ce public, l'influence des sources du sentiment d'auto-efficacité varie selon la personne, le contexte ou l'événement en présence (Mukamutara, 2012). Hoy et Spero (2005) insistent sur la distinction à établir entre sentiment d'efficacité personnelle des enseignants et sentiment d'efficacité général, ce dernier reflétant plutôt une croyance générale quant au pouvoir de l'enseignement sur l'éducation des enfants; ces deux construits étant depuis longtemps connus comme statistiquement indépendants (Gibson et Dembo, 1984). Le sentiment d'efficacité personnelle des enseignants est positivement lié à leur enthousiasme pour l'enseignement, à leur investissement dans la profession durant leurs premières années de carrière (Rots, Aelterman, Devos et Vlerick, 2010) ainsi qu'à la perception d'adéquation de leur formation initiale (Darling-Hammond, Chung et Frelow, 2002). En formation, le feedback évaluatif est particulièrement déterminant pour les croyances d'efficacité des apprenants (Galand et Vanlede, 2004). Plus largement, l'accompagnement (de stage ou mentoral) est, lui aussi, directement lié au sentiment d'auto-efficacité des étudiants/enseignants débutants, favorisant les expériences de maîtrise, la persuasion verbale et les situations d'apprentissage vicariant (Rots et al., 2010; Tschannen-Moran et Woolfolk-Hoy, 2007). 
Rots, Kelchtermans et Aelterman (2012) rappellent que les succès professionnels perçus comme les plus influents par les enseignants ont trait à l'expérience d'efficacité, au sentiment de faire la différence tant en tant que personne qu'en tant qu'enseignant auprès des élèves. Ils précisent que les élèves sont la principale référence (légitimante) à partir de laquelle les enseignants jugent leurs performances et justifient leur perception de soi en tant qu'enseignants. Enfin, ils ajoutent que la reconnaissance par des autres significatifs (e.g. collègues, mentors) constitue également une source essentielle d'affirmation de soi en tant que professionnel compétent chez les enseignants novices. Sachant que ces derniers, comme leurs collègues en cours de formation, présentent généralement un sentiment d'efficacité personnelle moins élevé que les enseignants expérimentés (Tschannen-Moran et Woolfolk-Hoy, 2007), et dans la mesure où le bagage des pompiers candidats aux modules pédagogiques varie fortement, nous considérerons avec attention ce point.

Cette synthèse met en lumière que le sentiment d'auto-efficacité des pompiers en matière de formation mérite toute notre attention. Disposer de données probantes et contextualisées à ce sujet est d'autant plus capital que la formation initiale des enseignants et celle des instructeurs pompiers divergent sur de nombreux plans, rendant les comparaisons ou inférences hasardeuses.

Dans cet article, nous tâcherons de répondre à une question principale (Q2) et à deux questions secondaires (Q1, Q3) se rapportant au sentiment d'auto-efficacité des pompiers en matière de formation. Précisons que le recours à la notion d'impact se justifie par le fait qu'à l'heure actuelle, les formations pédagogiques ne visent pas explicitement à accroitre le sentiment d'auto-efficacité pédagogique des pompiers belges. Si une variation du niveau de sentiment d'auto-efficacité devait ainsi être constatée entre l'entrée en formation pédagogique des agents et leur sortie, celle-ci ne pourrait être qualifiée d'effet à proprement parler.

Q1. La structure factorielle du questionnaire utilisé pour évaluer le sentiment d'auto-efficacité pédagogique des pompiers concorde-t-elle avec celle de l'outil dont il est dérivé et qui est propre à l'enseignement?

Q2. La formation pédagogique a-t-elle un impact sur le développement du sentiment d'autoefficacité des pompiers en matière de formation?

Q3. Le sentiment d'auto-efficacité se différencie-t-il selon le module pédagogique suivi par les candidats, selon leur expérience pédagogique préalable ou selon l'instructeur responsable de leur formation?

\section{Méthodologie}

Notre étude quantitative repose sur un dispositif de type pré-test/post-test instrumenté à l'aide d'un questionnaire rempli en classe. Un premier recueil de données a été effectué à l'entrée des candidats en formation (durant l'introduction du cours) et un deuxième à leur sortie (avant communication des résultats afin de ne pas biaiser les résultats). La procédure de collecte faisait partie intégrante du dispositif de formation et garantissait l'anonymat aux répondants. En général, six semaines s'écoulaient entre les pré- et post-tests, la formation s'organisant à raison d'un à deux jour(s) par semaine avec une pause de deux semaines entre les quatrième et cinquième jours (ce dernier étant dédié à la principale épreuve certificative du module). 


\section{Échantillon}

Notre échantillon se compose de 125 pompiers $^{4}$ (répartis en dix-huit groupes) ayant terminé l'un des modules de formation pédagogique reconnus par le Centre fédéral de Connaissance pour la Sécurité Civile (KCCE) entre 2015 et 2019 au sein du centre de formation de la province de Hainaut. Parmi eux, 90 (72,00 \%) ont suivi le FOROP1 dans sa nouvelle mouture (post-réforme), 27 (21,60 \%) ont suivi le module Instructeur du brevet d'Officier par promotion (pré-réforme) et 8 (6,40\%) ont suivi ce même module dans le cadre de leur brevet d'Officier par recrutement (pré-réforme). Par ordre croissant de grade, notre échantillon comporte 47 Sapeurs (37,60 \%), 36 Caporaux (28,80 \%), 23 Sergents (18,40 \%), 8 Adjudants (6,40 \%), 1 Lieutenant et 10 Sous-Lieutenants ou Capitaines stagiaires ${ }^{5}$. En matière d'ancienneté $34,40 \%$ sont pompiers depuis moins de dix ans, 42,40 \% exercent depuis dix à vingt ans et 23,20\% depuis plus de vingt ans. À leur entrée en formation, 65,60\% des candidats ( $\mathrm{n}=$ 82) ont déclaré disposer d'une expérience pédagogique. Pour plus d'un tiers $(39,00 \%)$, cette expérience provient de formations assurées en caserne. Vient ensuite la dispense de formations au sein de l'un des onze centres agréés en Belgique (23,20\%). On retrouve en troisième position les pompiers exerçant une activité complémentaire d'instructeur dans un organisme privé (14,60\%). Les autres expériences se partagent entre les activités de moniteur sportif (9,80\%), d'instructeur en aide médicale urgente $(2,40 \%)$ et d'enseignant $(2,40 \%)$. Viennent enfin les activités éducatives non spécifiées $(8,50 \%)$.

\section{Outil}

Notre outil consiste en un questionnaire papier auto-administré comportant seize items. Douze ont été rédigés en référence aux objectifs pédagogiques des modules de formation FOROP1 et Instructeur tels que définis par le législateur, auxquels ont été adjoints deux items portant sur la réflexivité et le perfectionnement professionnel (items 7 et 15) ainsi que deux items relatifs à l'intelligence émotionnelle et sociale (items 11 et 15) en raison de leur importance pour l'exercice de la fonction d'instructeur. Les items se présentent sous la forme d'affirmations ( Je me sens capable...») par rapport auxquelles les répondants doivent se positionner. Notre questionnaire s'inspire de la Teachers'Sense of Efficacy Scale (long form, TSES) de Tschannen-Moran et Woolfolk-Hoy (2001), traduit et validé en français auprès d'un public d'étudiants en enseignement (De Stercke, Temperman, De Lièvre et Lacocque, 2014). Néanmoins, il possède des particularités : 1) il repose sur une échelle métrique allant de 0 (Pas du tout d'accord) à 10 (Tout à fait d'accord) plutôt que sur une échelle de 1 à 9;2) comporte 16 items au lieu de 24 ; 3) ses items ne sont pas classés selon les trois sous-échelles de la TSES. Ces choix s'expliquent par les besoins de nos analyses ainsi que par les spécificités du contexte qui nous occupe. Tout comme la TSES, notre outil prévoit le calcul d'un score global de sentiment d'auto-efficacité sur la base de la moyenne générale non pondérée des items. 


\section{Résultats}

\section{Structure factorielle du questionnaire (Q1)}

Afin de répondre à notre première question de recherche, nous avons examiné la structure factorielle de notre questionnaire. Les analyses de fiabilité par consistance interne ont été conduites en prenant en compte ses 16 items. L'alpha de Cronbach obtenu sur base du pré-test (n valide $=122$ ) et du post-test (n valide $=123$ ) est de, 95. Ce score confirme que nos variables permettent d'évaluer un même construit. Le schéma directeur de notre questionnaire s'écartant de celui du TSES, une analyse en composantes principales avec rotation Varimax et normalisation de Kaiser a été effectuée à partir des résultats au pré-test afin d'explorer ses éventuelles dimensions. Il en ressort une structure en trois composantes après six rotations ${ }^{6}$. Le pourcentage de variance totale expliquée par ces trois facteurs cumulés s'élève à 70,57 \%, dont 56,39\% de valeur propre initiale pour le seul premier facteur. La rupture du coude de l'éboulis de Catell se situant au niveau de la deuxième composante, bien que le troisième facteur possède une valeur propre initiale supérieure à 1,000, il a été décidé de l'abandonner. L'analyse réalisée à nouveau sur cette base ${ }^{7}$ fait ressortir un modèle à deux composantes expliquant 64,32 \% de la variance totale. Dans la mesure où 56,39 \% de cette variance totale est expliquée par le premier facteur (soit neuf items expliqués), l'apport du deuxième facteur au modèle (un seul item expliqué) est négligeable. De surcroît, en analysant la saturation des items sur chacun des deux facteurs révélés par l'ACP après rotation, il s'avère impossible d'identifier une solution acceptable pour justifier leur distinction, et ce, en dépit de l'extraction des variables de réflexivité, de perfectionnement professionnel ou d'intelligence émotionnelle et sociale, par exemple. L'analyse répliquée à partir des données du post-test arrive aux mêmes conclusions. Ces observations indiquent que, contrairement à la TSES ou à son adaptation francophone, notre questionnaire possède une structure unifactorielle.

\section{Analyses descriptives (Q2)}

Intéressons-nous à présent à l'impact de la formation pédagogique sur le sentiment d'auto-efficacité des pompiers en matière de formation. Le tableau 1 expose la moyenne globale de sentiment d'autoefficacité pédagogique de nos sujets au pré-test (a), au post-test (b) et pour ces deux temps de mesure considérés ensemble (c). Le minimum, le maximum, l'écart-type et le coefficient de variation de ces trois variables sont également renseignés.

\section{Tableau 1}

Moyennes globales de SEP en formation $(n=125)$

\begin{tabular}{|l|c|c|c|c|c|}
\hline & Min & Max & Moy & E-T & CV (\%) \\
\hline a. Moyenne globale SEP (Pré-test) & 1,94 & 10,00 & 5,91 & 1,48 &, 25 \\
\hline b. Moyenne globale SEP (Post-test) & 4,88 & 9,81 & 7,89 &, 89 &, 11 \\
\hline c. Moyenne globale SEP (Pré-test + Post-test) & 4,25 & 9,59 & 6,90 & 1,00 &, 14 \\
\hline
\end{tabular}


La moyenne globale au pré-test est de 5,91 sur 10 et la moyenne globale au post-test de 7.89 sur 10, leurs écarts-types étant respectivement de 1,48 et ,89. Un gain relatif a été calculé à partir de ces moyennes, conformément à la formule : (Score APRES - Score AVANT) / (Score MAXIMUM - Score AVANT) *100. L'effet d'apprentissage étant généralement admis à partir du seuil des $30 \%$ (D'Hainaut, 1975), le gain relatif moyen (perçu) obtenu présentement, soit $48.41 \%$, atteste de l'impact de la formation pédagogique sur le développement du sentiment d'auto-efficacité des candidats. Cette conclusion est corroborée par le test $t$ de Student qui révèle la différence statistiquement significative entre les moyennes du pré-test et du post-test $(t=15,904 ; \alpha<, 000)$. Le coefficient de variation est plus de deux fois moins élevé à la sortie qu'à l'entrée en formation, attestant de l'effet d'équité du dispositif. Enfin, la corrélation entre le score au pré-test et le gain relatif permet d'affirmer que les candidats se sentant les moins efficaces au début de la formation pédagogique sont ceux qui progressent le plus en la matière au cours de celle-ci et inversement ( $\mathrm{n}$ valide $=124 ; \mathrm{r}=-, 424 ; \alpha<, 01$ ).

Le tableau 2 présente les moyennes au pré-test et au post-test ainsi que le gain brut et le gain relatif moyens constatés entre ces deux temps de mesure pour les seize variables du questionnaire.

Tableau 2

Moyennes aux pré-test et post-test, gain et gain relatif moyens

1. Expliquer les notions-clefs de la pédagogie et de la communication

2. Motiver les candidats à apprendre

3. Poser et faire respecter un cadre disciplinaire propice à l'apprentissage

4. Appliquer les différentes méthodes pédagogiques pour favoriser l'apprentissage des candidats

5. Mettre en œuvre l'évaluation formative

6. Concevoir un scénario/une fiche pédagogique en m'assurant de la cohérence entre les objectifs, les méthodes et l'évaluation

7. Prendre de la distance par rapport à mes pratiques pédagogiques en vue de les améliorer

8. Différencier les types d'objectifs pédagogiques

9. Ajuster mon cours au profil des candidats à former (expérience, grade/ fonction, zone de secours...)

10. Animer un cours sur base d'un scénario/d'une fiche pédagogique 11. Percevoir, exprimer, comprendre, gérer et utiliser à bon escient mes émotions en situation de formation

12. Conduire un briefing et un débriefing

13. Utiliser les supports/outils technopédagogiques

14. Préparer et corriger des questions d'examen

15. Continuer à me former dans le domaine de la pédagogie afin de perfectionner mes pratiques

16. Reconnaître et comprendre les émotions des candidats afin d'interagir positivement avec eux et de les aider à les gérer

\begin{tabular}{cccc}
$\begin{array}{c}\text { Moy } \\
\text { Pré-test }\end{array}$ & $\begin{array}{c}\text { Moy } \\
\text { Post-test }\end{array}$ & Gain & GR (\%) \\
4,34 & 7,36 & $+3,02$ & 53,36 \\
6,50 & 7,99 & $+1,49$ & 42,57 \\
6,49 & 8,18 & $+1,69$ & 48,15 \\
4,86 & 7,74 & $+2,88$ & 56,03 \\
& & & \\
5,06 & 7,66 & $+2,60$ & 52,63 \\
5,15 & 7,75 & $+2,60$ & 53,61 \\
& & & \\
6,86 & 8,30 & $+1,44$ & 45,86 \\
& & & \\
5,05 & 7,59 & $+2,54$ & 51,31 \\
6,45 & 8,20 & $+1,75$ & 49,30 \\
& & & \\
6,04 & 8,04 & $+2,00$ & 50,51 \\
6,09 & 7,62 & $+1,53$ & 39,13 \\
& & & \\
6,08 & 8,07 & $+1,99$ & 50,76 \\
\hline 5,85 & 7,94 & $+2,09$ & 50,36 \\
6,13 & 7,46 & $+1,33$ & 34,36 \\
7,36 & 8,31 &,+ 95 & 35,98 \\
& & & \\
6,30 & 8,01 & $+1,71$ & 46,22 \\
& & & \\
& & & \\
\hline
\end{tabular}


La moyenne la plus basse au pré-test est celle de l'item $1(4,34)$ et la moyenne la plus haute celle de l'item $15(7,36)$. Au post-test, ce sont à nouveau ces items 1 et 15 qui possèdent respectivement les moyennes la plus basse $(7,36)$ et la plus haute $(8,31)$. On note à chaque fois une progression des moyennes des variables entre les pré-test et post-test (gain) qui se traduit tout aussi systématiquement par un effet d'apprentissage (gain relatif). L'effet d'apprentissage le plus marqué est observé au niveau de l'item 4 (56,03\%), suivi des items $6(53,61 \%)$ et 1 (53,36 \%). Le plus faible se situe au niveau de l'item 14 (34,36\%). Ces scores sont cohérents avec la finalité du FOROP1; qui, s'il aborde les notions d'évaluation, n'octroie pas de temps à la rédaction/correction de questions d'examen. Le module Instructeur des brevets d'Officier par promotion et d'Officier par recrutement comporte quant à lui une activité sur le sujet. De fait, les candidats au brevet d'Officiers par promotion progressent plus dans ce domaine ( $\mathrm{GR}=40,46 \%)$ que leurs collègues candidats au FOROP1 ( $\mathrm{GR}=34,00 \%)$. Étrangement, le sentiment d'auto-efficacité de ces derniers progresse plus que celui des candidats au brevet d'Officier par recrutement pour cette variable $(\mathrm{GR}=15,75 \%)$.

Quel que soit le module, de nombreuses séquences sont dédiées à la découverte et à la mise en œuvre des méthodes pédagogiques en classe et sur le terrain. Ceci explique la progression importante constatée pour l'item 4. L'item 6 renvoie à la tâche pré-active conditionnant les activités de micro-enseignement formative (jour 4) et certificative (jour 5) qui sont au cœur de la formation. Une demi-journée de travail supervisé est consacrée à cette tâche, complétée par des échanges à distance. Il n'est donc pas étonnant que les candidats aient le sentiment d'avoir fortement progressé en la matière durant la formation.

\section{Analyses corrélationnelles}

Afin de poser les bases de nos analyses inférentielles, observons les relations entre les variables de notre questionnaire. Les résultats du pré-test (tableau 3) indiquent que celles-ci sont toutes positivement corrélées, selon des intensités relativement faibles, modérées à fortes. Les deux corrélations les moins importantes (items $1 \mathrm{x} 7$ et items $7 \mathrm{x} 13$ ) sont également les seules à être significatives à $\alpha<, 05$. Les autres sont toutes significatives à $\alpha<.01$.

\section{Tableau 3}

Corrélations entre les variables pour le pré-test $(n=122)$

\begin{tabular}{|c|c|c|c|c|c|c|c|c|c|c|c|c|c|c|c|c|}
\hline Var. & 1 & 2 & 3 & 4 & 5 & 6 & 7 & 8 & 9 & 10 & 11 & 12 & 13 & 14 & 15 & 16 \\
\hline 1 & 1 & & & & & & & & & & & & & & & \\
\hline 2 &, $543^{* *}$ & 1 & & & & & & & & & & & & & & \\
\hline 3 &, $494^{* *}$ &, $678^{* *}$ & 1 & & & & & & & & & & & & & \\
\hline 4 &, $660^{* *}$ & ,591** &, $541^{* *}$ & 1 & & & & & & & & & & & & \\
\hline 5 &, $670^{* *}$ &, $544^{* *}$ &, $481^{* *}$ &, $744^{* *}$ & 1 & & & & & & & & & & & \\
\hline 6 &, $587^{* *}$ &, $543^{* *}$ &, $504^{* *}$ &, $7111^{* *}$ &, $749^{* *}$ & 1 & & & & & & & & & & \\
\hline 7 &, $228^{*}$ &, $476^{* *}$ &, $451^{* *}$ &, $346^{* *}$ &, $347^{* *}$ &, $395^{* *}$ & 1 & & & & & & & & & \\
\hline 8 &, $675^{* *}$ &, $545^{* *}$ &, $404^{* *}$ &, $634^{* *}$ &, $755^{* *}$ &, $694^{* *}$ & $464^{* *}$ & 1 & & & & & & & & \\
\hline 9 & $462^{* *}$ &, $605^{* *}$ &, $505^{* *}$ &, $560^{* *}$ &, $553^{* *}$ &, $587^{* *}$ &, $554^{* *}$ &, $661^{* *}$ & 1 & & & & & & & \\
\hline 10 &, $582^{* *}$ &, $630^{* *}$ &, $657^{* *}$ &, $618^{* *}$ &, $624^{* *}$ &, $672^{* *}$ &, $426^{* *}$ &, $609^{* *}$ &, $772^{* *}$ & 1 & & & & & & \\
\hline 11 &, $426^{* *}$ & , $597^{* *}$ &, $610^{* *}$ &, $575^{* *}$ &, $543^{* *}$ &, $607^{* *}$ &, $349^{* *}$ &, $451^{* *}$ &, $643^{* *}$ &, $723^{* *}$ & 1 & & & & & \\
\hline 12 &, $512^{* *}$ &, $545^{* *}$ &, $630^{* *}$ &, $524^{* *}$ &, $535^{* *}$ &, $517^{* *}$ &, $398^{* *}$ &, $538^{* *}$ &, $622^{* *}$ &, $673^{* *}$ &, $609^{* *}$ & 1 & & & & \\
\hline 13 &, $486^{* *}$ & , 339** &, $371^{* *}$ &, $396^{* *}$ &, $504^{* *}$ &, $520^{* *}$ &, $212^{*}$ &, $436^{* *}$ &, $504^{* *}$ &, $589^{* *}$ &, $520^{* *}$ &, $579^{* *}$ & 1 & & & \\
\hline 14 &, $386^{* *}$ & ,427** &, $491^{* *}$ &, $4222^{* *}$ &, $369^{* *}$ &, $477^{* *}$ &, $342^{* *}$ &, $415^{* *}$ &, $607^{* *}$ &, $604^{* *}$ &, $526^{* *}$ &, $630^{* *}$ &, $492^{* *}$ & 1 & & \\
\hline 15 &, $291^{* *}$ &, $485^{* *}$ &, $511^{* *}$ &, $361^{* *}$ &, $380^{* *}$ &, $472^{* *}$ &, $549^{* *}$ &, $400^{* *}$ &, $428^{* *}$ &, $544^{* *}$ &, $447^{* *}$ &, $353^{* *}$ &, $324^{* *}$ &, $435^{* *}$ & 1 & \\
\hline 16 &, $479^{* *}$ &, $653^{* *}$ &, $556^{* *}$ &, $615^{* *}$ &, $587^{* * *}$ &, $583^{* *}$ &, $521^{* *}$ &, $613^{* *}$ &, $708^{* *}$ & ,696** & ,695** &, $562^{* *}$ &, $463^{* *}$ &, $563^{* *}$ &, $559^{* *}$ & 1 \\
\hline & & & & & & & & & & & & & & & ${ }^{* *} a<$, & $a<$, \\
\hline
\end{tabular}

8 - Formation et profession 29(2), 2021 
En répliquant cette analyse au niveau du post-test, on arrive à la même conclusion : les corrélations sont positives, relativement faibles à modérées ou fortes; toutes significatives à $\nabla<, 01$. La plus faible est celle unissant les items 1 et 7 (,263), qui étaient déjà les moins corrélés du pré-test (,228). La corrélation entre les items 7 et 13, qui était de ,212 au pré-test passe quant à elle à ,478 au post-test.

\section{Analyses inférentielles (Q3)}

Adoptons à présent une démarche comparative. Tout d'abord, voyons si les moyennes de sentiment d'auto-efficacité du pré-test et du post-test sont différentes selon le module auquel étaient inscrits les candidats. En ce qui concerne le pré-test, une différence significative est observée entre les groupes $(\mathrm{F}=6,979 ; \mathrm{ddl}=2 ; \alpha<, 01)$ en faveur des candidats du module Instructeur du brevet d'Officier par promotion. Ces derniers affichent une moyenne de 6,68 contre 5,78 pour les candidats du module FOROP1 et 4,76 pour les candidats du module Instructeur du brevet d'Officier par recrutement. Cette différence significative en faveur des candidats Officiers par promotion continue de se marquer au post-test $(\mathrm{F}=5,688 ; \mathrm{ddl}=2 ; \alpha<, 01)$, bien que les écarts entre les moyennes de chaque groupe se réduisent ( $\mathrm{x}$ mod. Instructeur Of. promo. =8,31; $\mathrm{x}$ mod. Instructeur Of. recrut. =7,26; $\mathrm{x}$ FOROP1 = $7,82)$. Que ce soit aux temps 1 ou 2, aucune différence significative n'est relevée entre les candidats du brevet d'Officier par recrutement et les candidats du FOROP1.

Ensuite, analysons si les candidats déclarant avoir de l'expérience pédagogique à l'entrée du module $(\mathrm{n}=82)$ affichent des moyennes de sentiment d'auto-efficacité plus élevées que leurs collègues $(\mathrm{n}=$ 43) avant et après la formation. Au pré-test, une différence statistiquement significative est relevée en faveur des pompiers possédant une expérience pédagogique antérieure (x SEP global expérimentés = $6,24$ vs $\mathrm{x}$ SEP global novices = 5,29; F = 12,541; ddl $=1 ; \alpha<, 01)$. En revanche, cette différence ne s'exprime plus au post-test ( $\mathrm{x}$ SEP global expérimentés $=7,98$ vs $\mathrm{x}$ SEP global novices $=7,71 ; \mathrm{F}=$ $2,780 ; \mathrm{ddl}=1 ; \alpha<, 10)$, ce qui confirme l'effet lissant de la formation pédagogique sur les croyances d'efficacité des pompiers.

Enfin, prêtons attention aux instructeurs responsables des formations pédagogiques. Bien qu'ils soient au nombre de quatre, aucune différence significative n'est constatée entre les moyennes globales de sentiment d'auto-efficacité au post-test des candidats $(\mathrm{F}=1,346 ; \mathrm{ddl}=3 ; \alpha=, 263)$.

\section{Discussion}

Tâchons d'interpréter les résultats liés à nos questions de recherche 1 et 3 . En ce qui concerne la divergence entre la structure unifactorielle de notre questionnaire et celle, multifactorielle, de la TSES, nous pensons que les représentations sociales et l'état de l'art dans les domaines de la formation des professionnels de la sécurité civile et de l'enseignement sont en partie en cause. Si le schéma directeur de la TSES traduit les grands champs d'action de l'enseignant, largement documentés, les items de notre outil ont pour rappel été rédigés en référence aux objectifs des formations pédagogiques accessibles aux pompiers. Ces objectifs, bien que cohérents avec les descriptions de fonction ministérielles associées aux fonctions d'instructeurs (FOROP1) et de concepteur/manager de formation (FOROP2), ne s'enracinent pas directement dans la réalité de ces dernières, en omettant certains aspects (la gestion de la logistique, par exemple). En interrogeant l'activité «réelle» des instructeurs pompiers, il serait 
certainement possible d'actualiser ces objectifs, de les compléter, et par la même occasion d'améliorer non seulement la pertinence de la formation pédagogique, mais également de bonifier le questionnaire utilisé dans le cadre de cette recherche. Peut-être alors sa structure factorielle s'en trouvera-t-elle changée. On peut voir là une limite de cette recherche qui, en explorant les contours d'un construit encore peu défini dans le contexte considéré, souffre nécessairement de certains manquements méthodologiques en plus de sa nature écologique.

En ce qui concerne nos analyses comparatives, la différence entre les moyennes des deux groupes inscrits au module Instructeur, d'une part les candidats Officiers par promotion et d'autre par les candidats Officiers par recrutement, qui ont suivi la même formation, pourrait s'expliquer par le fait que les premiers accusaient au moment de celle-ci plusieurs années d'expérience en tant que pompier, voire en tant qu'instructeur, les seconds n'ayant quant à eux aucune expérience acquise dans ces domaines. Considérant la littérature sur les facteurs propices au développement du sentiment d'auto-efficacité, tant de manière générale qu'en enseignement, il est évident que disposer d'un bagage d'instructeur, même réduit, augmente les chances d'avoir vécu des expériences actives de maitrise en formation, d'avoir bénéficié de persuasion verbale (via rétroaction d'un membre de la cellule pédagogique, par exemple) ou fait des expériences vicariantes (par comparaison sociale ou modelage à l'occasion de formations assurées avec un pair). Ce résultat corrobore l'étude de Pfitzner-Eden (2016) qui met en évidence le lien positif et étroit entre les expériences antérieures de maîtrise et le sentiment d'autoefficacité chez les enseignants. Rappelons-nous les différences de sentiment d'auto-efficacité mises au jour entre enseignants novices et enseignants expérimentés, en faveur de ces derniers, et l'influence de l'accompagnement sur l'accroissement du sentiment d'efficacité personnelle des enseignants (Rots et al., 2010; Tschannen-Moran et Woolfolk-Hoy, 2007). Étant donné les normes d'encadrement strictes des formations pratiques chez les pompiers, il est rare qu'un instructeur débutant se retrouve seul face à son groupe et à ses responsabilités en début de «carrière». Lorsqu'on ajoute à cela la supervision et le coaching offerts par la cellule pédagogique de la plupart des centres de formation, on pourrait s'attendre à ce que le sentiment d'isolement vécu par de nombreux enseignants débutants (Mukamurera, 2011) ne soit pas ressenti avec autant d'intensité par les instructeurs pompiers novices.

Les différences significatives constatées entre le sentiment d'auto-efficacité des candidats du FOROP1 et des candidats du brevet d'Officier par promotion sont difficiles à expliquer. Une piste serait que ces derniers ont suivi avec succès davantage de formations de base que les pompiers inscrits au FOROP1 et qu'ils sont globalement plus gradés (deux fois plus de Sous-Officiers et trois Officiers contre aucun chez les candidats FOROP1). Une autre hypothèse serait qu'ils sont plus nombreux à déclarer avoir de l'expérience pédagogique à l'entrée de la formation (81,48 \% contre 63,33\% chez les candidats au FOROP1 et 37,50 \% chez les candidats au brevet d'Officier par recrutement). Restons toutefois prudents vu les effectifs des groupes. 
L'absence de différence significative au post-test entre les apprenants selon qu'ils aient suivi leur formation pédagogique avec l'un des quatre instructeurs accrédités à la dispenser pourrait s'expliquer par l'harmonisation de leurs pratiques, du dispositif et des supports de formation. Cette logique d'efficience est en effet fortement soutenue tant par les instances fédérales (KCCE) que par l'école du feu dans laquelle ils exercent.

Enfin, on comprend mieux la progression du sentiment d'auto-efficacité des pompiers au cours de leur formation pédagogique lorsqu'on sait que le dispositif sur lequel elle s'appuie met en œuvre de nombreux principes propices à un enseignement efficace. Il imprime une démarche explicite et systématique (Hattie,2009), graduelle et engageante (Galand et Vanlede, 2004), recourt à la formulation claire des objectifs en début de formation et à leur rappel selon les tâches à accomplir (Mc Crudden, Magliano et Schraw, 2010), et fait fréquemment usage du feedback spécifique, commenté et centré sur le progrès (Hattie, 2009). Quand on ajoute à cela l'énergie déployée par les instructeurs pour établir un environnement d'apprentissage optimal en classe (Heutte, 2017), le taux de réussite de notre échantillon à l'issue de sa formation (98,40 \%) s'entend parfaitement.

\section{Conclusion}

Cette étude établit non seulement que la formation pédagogique a un impact positif sur le sentiment d'auto-efficacité des pompiers en matière de formation (effet d'apprentissage), mais qu'elle réduit également les disparités entre les croyances d'efficacité des apprenants (effet d'équité). Elle montre par ailleurs que la structure factorielle de notre questionnaire est unidimensionnelle, au contraire de la TSES ou de son adaptation francophone. À ce propos, nous pouvons estimer que notre outil produit une mesure pertinente pour évaluer l'impact, voire l'effet, de la formation pédagogique des pompiers tout en constituant un levier intéressant pour stimuler leur réflexivité et leur agentivité. Nos analyses mettent en lumière que les apprenants plus formés, plus expérimentés à l'opérationnel ou possédant une expérience pédagogique antérieure ont un sentiment d'auto-efficacité plus élevé que leurs collègues, cette dernière tendance disparaissant au post-test. Enfin, le sentiment d'auto-efficacité des candidats au terme de leur formation n'est pas influencé par l'instructeur qui les avait en charge. Ces résultats qui posent les bases de la recherche sur le sentiment d'auto-efficacité pédagogique des pompiers ouvrent la voie à nombre d'investigations complémentaires. Elles appellent notamment à la conduite de recherches qualitatives visant à cerner la réalité des fonctions d'instructeurs (FOROP1) et de concepteur (FOROP2) afin d'améliorer les formations y menant. La complexité de ces fonctions pose par ailleurs la question de la professionnalisation des pompiers s'orientant vers la formation comme domaine de spécialisation; domaine qui, espérons-le, sera promptement reconnu à sa juste valeur dans notre pays. 


\section{Notes}

1 Au nombre de 35 en Belgique, ces zones s'organisent en un réseau de postes de secours et sont dirigées par un Commandant de zone (Loi relative à la sécurité civile, 2007).

2 Le M01 vise l'obtention du brevet de Sergent (premier niveau de Sous-Officier) et l'OFF2 celle du brevet de Capitaine (deuxième niveau d'Officier).

3 En 2020, 224 instructeurs étaient en activité à l'école du feu du Hainaut, pour un total de 1.859 agents au sein des trois zones de secours du territoire provincial. Les instructeurs issus d'autres provinces étant en nombre restreint au sein de ce pool, il semble acceptable d'affirmer qu'à peu près $10 \%$ des pompiers hennuyers sont instructeurs.

4 Pour quelque 17.000 pompiers en Belgique (KCCE, 2017).

5 Ces deux grades étant les grades de recrutement des Officiers, respectivement avant et après la réforme.

6 L'indice KMO de ,92 atteste de la pertinence de l'analyse factorielle et le test de Bartlett indique une corrélation suffisante des variables entre elles (khi-carré approximé $=1438 ; \mathrm{ddl}=2 ; \nabla=, 000$ ), sans qu'une situation de sphéricité ne soit constatée. La condition de non-colinéarité est par ailleurs rencontrée puisque le déterminant de la matrice de corrélation est supérieur à ,0001.

7 Celle-ci respecte les conditions du KMO, du test de Bartlett et de non-colinéarité.

8 Ceci ne signifie pas que sur le plan individuel aucune régression n'a lieu entre le pré-test et le post-test.

\section{Références}

Arrêté ministériel relatif à la formation et au certificat FOROP1 et FOROP2 pour les membres des services publics de secours (2019). Moniteur belge, 12 avril.

Arrêté royal relatif à la formation des membres des services publics de secours et modifiant divers arrêtés royaux (2015). Moniteur belge, 7 décembre.

Bandura, A. (2003). Auto-efficacité : Le sentiment d'efficacité personnelle. Bruxelles : De Boeck.

D’Hainaut, L. (1975). Concepts et méthodes de la statistique. Tome 1. Bruxelles : Labor.

Darling-Hammond, L., Chung, R., et Frelow, F. (2002). Variation in teacher preparation: How well do different pathways prepare teachers to teach? Journal of Teacher Education, 53(4), 286-302. https://doi.org/10.1177/0022487102053004002

De Stercke, J., Temperman, G., De Lièvre, B., et Lacocque, J. (2014). Échelle de sentiment d'efficacité personnelle : traduction francophone de la Teachers'Sense of Efficacy Scale. Service de Pédagogie Générale et des Médias Éducatifs, Université de Mons. En ligne : http://bit.do/TSESfr [Consulté le 02/07/2021].

Deppa, K.F., et Saltzberg, J. (2016). Resilience Training for Firefighters. An Approach to prevent Behavioral Health Problems. SpringerBriefs in Fire: Cham, Switzerland. https://doi.org/10.1007/978-3-319-38779-6

Galand, B., et Vanlede, M. (2004). Le sentiment d'efficacité personnelle dans l'apprentissage et la formation : Quel rôle joue-t-il? D'où vient-il? Comment intervenir? Savoirs, 5, 91-116.https://doi.org/10.3917/savo.hs01.0091

Gibson, S., et Dembo, M. (1984). Teacher efficacy: a construct validation. Journal of Educational Psychology, 76(4), $569-582$. https://doi.org/10.1037/0022-0663.76.4.569

Hattie, J.A. (2009). Visible Learning: A Synthesis of Over 800 Meta-Analyses Relating to Achievement. New York : Routledge.

Heutte, J. (2017). L'environnement optimal d'apprentissage tout au long et tout au large de la vie : Contribution de la recherche empirique sur les déterminants psychologiques de l'expérience positive subjective aux sciences de l'éducation et de la formation des adultes. Sciences et Bonheur, 2, 82-99. https://hal.archives-ouvertes.fr/hal-01597551 
Hoy, A., et Spero, R. (2005). Changes in teacher efficacy during the early years of teaching: A comparison of four measures. Teaching and Teacher Education, 21(4), 343-356. https://doi.org/10.1016/j.tate.2005.01.007

KCCE (2017). Le personnel des pompiers en Belgique. Bruxelles : SPF Intérieur. https://www.civil-security.be/fr/content/que-font-les-pompiers

Lambert, J.E., Benight, C.C., Harrison, E., et Cieslak, R. (2012). The Firefighter Coping Self-Efficacy Scale: measure development and validation. Anxiety, Stress and Coping, 25(1), 79-91. https://doi.org/10.1080/10615806.2011.567328

Loi du 15 mai 2007 relative à la sécurité civile (2007). Moniteur belge, 31 juillet.

Marka-Studzinska, M., Golonka, K., et Izydorczyk, B. (2019). Self-Efficacy as a Moderator between Stress and Professional Burnout in Firefighters. International Journal of Environmental Research and Public Health, 16(2), 183. https://doi.org/10.3390/ijerph16020183

Mc Crudden, M.T., Magliano, J.P., et Schraw, G. (2010). Exploring how relevance instructions affect personal reading intentions, reading goals, and text processing: A mixed methods study. Contempary Educational Psychology, 35(4), 229241. https://doi.org/10.1016/j.cedpsych.2009.12.001

Mukamurera, J. (2011). Les multiples dimensions de l'insertion professionnelle : portrait, expériences et significations d'enseignants. Dans B. Wentzel, A. Akkari, P-F Coen et N. Changkakoti (dir.), Insertion professionnelle des enseignants : regards croisés et perspectives internationales. (pp. 17-39). Bejune : HEP.

Mukamutara, I. (2012). Le sentiment d'efficacité personnelle des enseignantes et des enseignants débutants du secondaire au Québec (Thèse de Doctorat). Université de Sherbrooke, Sherbrooke. En ligne : $\underline{\text { http://doc.rero.ch/record/235578 }}$

Pfitzner-Eden, F. (2016). Why do I feel more confident? Bandura's sources predict preservice' latent changes in teacher self-efficacy. Frontiers in psychology, 7:1486. https://doi.org/10.3389/fpsyg.2016.01486

Rots, I., Aelterman, A., Devos, G., et Vlerick, P. (2010). Teaching education and the choice to enter the teaching profession: A prospective study. Teaching and Teacher Education, 26(8), 1619-1629. https://doi.org/10.1016/j.tate.2010.06.013

Rots, I., Kelchtermans, G., et Aelterman, A. (2012). Learning (not) to become a teacher: A qualitative analysis of the job entrance issue. Teaching and Teacher Education, 28(1), 1-10. https://doi.org/10.1016/j.tate.2011.08.008

Tschannen-Moran, M., et Woolfolk-Hoy, A. (2001). Teacher efficacy: Capturing an elusive construct. Teaching and Teacher Education, 17(7), 783-805.

https://doi.org/10.1016/S0742-051X(01)00036-1

Tschannen-Moran, M., et Woolfolk-Hoy, A. (2007). The differential antecedents of self-efficacy beliefs of novice and experienced teachers. Teaching and Teachers Education, 23(6), 944-956. https://doi.org/10.1016/j.tate.2006.05.003

\section{Pour citer cet article}

De Stercke, J. et Temperman, G. (2021). Le sentiment d'auto-efficacité des pompiers en matière de formation. Formation et profession, 29(2), 1-13. http://dx.doi.org/10.18162/fp.2021.590 